\title{
Rising Trends in Medication Non-compliance and Associated Worsening Cardiovascular and Cerebrovascular Outcomes Among Hospitalized Adults Across the United States
}

\author{
Rupak Desai ${ }^{1}$, Samarthkumar Thakkar ${ }^{2}$, Hee Kong Fong ${ }^{3}$, Yash Varma ${ }^{4}$, Mir Z. Ali Khan ${ }^{5}$, Vikram B. \\ Itare $^{6}$, Jilmil S. Raina ${ }^{6}$, Sejal Savani ${ }^{7}$, Nanush Damarlapally ${ }^{8}$, Rajkumar P. Doshi ${ }^{9}$, Kishorbhai Gangani \\ 10 , Kranthi Sitammagari ${ }^{11}$ \\ 1. Cardiology, Atlanta Veterans Affairs Medical Center, Decatur, USA 2. Internal Medicine, Rochester General Hospital, \\ Rochester, USA 3. Cardiovascular Medicine, University of California Davis Medical Center, Sacramento, USA 4. Internal \\ Medicine, Government Medical College, Bhavnagar, IND 5. Internal Medicine, Saint Peters University Hospital, New \\ Brunswick, USA 6. Internal Medicine, Smolensk State Medical University, Smolensk, RUS 7. Public Health, New York \\ University, New York, USA 8. Health Sciences, Coleman College of Health Sciences, Houston, USA 9. Internal Medicine, \\ University of Nevada, Reno School of Medicine, Reno, USA 10. Internal Medicine, Texas Health Arlington Memorial \\ Hospital, Arlington, USA 11. Internal Medicine, Atrium Health Union, Monroe, USA
}

Corresponding author: Rupak Desai, drrupakdesai@gmail.com

\section{Abstract \\ Introduction}

Small-scale studies have described concerning rates of non-compliance/nonadherence towards groups of medications for primary and secondary prevention. Trends in cardiovascular and cerebrovascular events (CCE) among hospitalized patients with a non-compliant behavior towards medication, on the whole, remains unexplored on a large scale.

\section{Methods}

Using the National Inpatient Sample databases (2007-2014), we sought to assess the prevalence and trends in all-cause mortality and CCE in adult patients hospitalized with medication non-compliance. We compared baseline characteristics and comorbidities in the non-compliant patients with and without concomitant in-hospital CCE.

\section{Results}

We identified 7,453,831 adult hospitalizations with medication non-compliance from 2007 to 2014, of which 867,997 (11.6\%) patients demonstrated in-hospital CCE. Non-compliant patients with CCE consisted of a higher number of older, white, male patients having greater comorbid risk factors. Non-compliant

Received 07/29/2019

Review began $08 / 03 / 2019$ Review ended 08/04/2019 Published 08/14/2019

\section{() Copyright 2019}

Desai et al. This is an open access article distributed under the terms of the Creative Commons Attribution License CC-BY 3.0., which permits unrestricted use, distribution, and reproduction in any medium, provided the original author and source are credited. patients with CCE had higher all-cause in-hospital mortality ( $3 \%$ vs. $0.7 \%$ ), frequent transfers $[4.4 \%$ vs. $1.8 \%$ transfers to short-term hospitals, and $17.6 \%$ vs. $11.6 \%$ other transfers (skilled nursing or intermediate care facilities)], lower routine discharges (59.4\% vs. $71.1 \%$ ), and higher mean hospital charges ( $\$ 52,740$ vs. $\$ 30,748$ ) compared to non-compliant patients without CCE. Remarkably, this study demonstrates the rising trend in medication non-compliance across all age, sex, and race groups, and related in-hospital mortality, CCE, transfers to other facilities, and the health care cost from 2007 to 2014.

\section{Conclusions}

We observed rising trends in the prevalence of medication non-compliance and subsequent in-hospital mortality in hospitalizations among adults from 2007 to 2014. Non-compliant patients with inpatient CCE demonstrated rising trends in all-cause mortality, complications, health care utilization, and cost from 2007 to 2014 .

Categories: Cardiology, Internal Medicine, Epidemiology/Public Health

Keywords: mortality, medication non-compliance, non adherence, cardiovascular diseases, cerebrovascular disease, stroke, myocardial infarction, arrhythmias, venous thromboembolism, trends

\section{Introduction}

According to the World Health Organization, medication non-compliance or nonadherence has emerged as a major problem in developing countries where only $50 \%$ adherence has been found among patients with a long-term illness and the problem is expected to be greater in underdeveloped countries in view of the prevailing dearth of health care resources and disparities in health care delivery [1]. A meta-analysis of 376,162 patients established the positive effect of guideline-directed medical therapy (GDMT) for both the 
primary and secondary prevention of cardiovascular disease (CVD) and concluded that measures to improve overall adherence are desirable as opposed to class-specific adherence [2]. A meta-analysis of 21 studies revealed that GDMT can reduce the risk of all-cause mortality by $40 \%$ and cardiovascular events by $30 \%$ in patients with established CVD [3]. Similarly, a dose-response analysis discovered that higher compliance with antihypertensive medication was associated with a lower risk of both hemorrhagic and ischemic stroke [4]. Therefore, nonadherence to antihypertensive medications has shown to increase both cardiovascular and cerebrovascular events (CCE) and related mortality [5]. Multiple factors can contribute towards poor adherence which can be divided into socioeconomic, motivational, and communicational causes [6]. Earlier studies have evaluated the outcomes in the form of mortality and cost of care related to non-compliance to a group of medications mainly including antihypertensive, antidiabetics, or lipidlowering agents [7-9]. However, studies describing the sequential intersection of multiple sociodemographic and comorbid variables influencing medication non-compliance and cardiovascular outcomes would be crucial to perform as prior studies have called for improvement in overall patient adherence/compliance rather than just towards a class of medication [2]. Hence, using a nationally representative cohort from the National Inpatient Sample (NIS) databases (2007-2014), we postulate to reveal the rising prevalence and concerning trends in medication non-compliance, all-cause inpatient mortality, and CCE among patients with non-compliant behaviors irrespective of class of medications. We also compared characteristics and manifest comorbidities in non-compliant patients with and without incidental CCE.

\section{Materials And Methods \\ Data source}

This retrospective study analyzed the NIS databases (2007-2014), which is a division of the Healthcare Cost and Utilization Project, developed by the Agency for Healthcare Research and Quality. The NIS is the largest publicly available hospital discharge administrative database consisting of $20 \%$ stratified sampling of all inpatient admissions (more than seven million hospital stays per year) to non-federal hospitals in the United States. Considering the de-identified nature of the admission records, an approval from the institutional review board was not mandatory for this study.

\section{Study population and variables}

From January 2007 to December 2014, adult patients were identified with a non-compliant behavior towards medications using the International Classification of Diseases, Ninth Revision, Clinical Modification diagnostic code V15.81 [10]. These patients were divided into two study groups: those who experienced CCE vs. those who did not. We evaluated the frequency of various CCE which included first or subsequent myocardial infarction (MI), first or subsequent stroke, out of hospital cardiac arrest, arrhythmias, and venous thromboembolism (Appendix). The details of NIS data on variables, demographics, hospital-level characteristics, and comorbidities have been published previously [11-13]. We examined and compared the following baseline variables between the two groups: demographics, the source of payer and median household income quartile for patients' zip code, hospital-related variables, and pre-existing comorbidities.

\section{Primary and secondary outcomes}

The primary outcomes were defined as in-hospital mortality, disposition, and health care resource utilization in terms of mean length of stay (LOS, days) and hospital charges (USD). The secondary outcomes were the trends in all-cause in-hospital mortality and CCE in patients admitted with medication nonadherence regardless of medication group from 2007 to 2014.

\section{Statistical analyses}

Statistical analyses were performed using SPSS V.22.0 (IBM Corp., Armonk, NY, USA). Pearson's chi-square test is used for the categorical variables which were presented as numbers and proportions, and the Student t-test was used for the continuous variables which were presented as the mean and standard deviation. Furthermore, we stratified the population into the age groups of $18-44,45-64$, and $\geqslant 65$ years. A two-sided pvalue $\leqslant 0.05$ was defined as a statistical significance.

\section{Results}

From 2007 to 2014, we identified a total of 7,453,831 hospitalizations among the adult population with a history of medication nonadherence. Of these, 867,997 (11.6\%) patients experienced in-hospital CCE. Table 1 displays the patients and hospital-related characteristics as well as pre-existing comorbidities in nonadherent adults with and without CCE. Patients non-compliant to medications who experienced CCE consisted of higher number of older (mean age 59.6 vs. 51.0 years, $45-64$ years: $50.1 \%$ vs. $43.8 \%$ ), white (58.0\% vs. $50.5 \%)$, male (62.1\% vs. $37.9 \%)$ patients. The CCE cohort more often consisted of Medicare enrollees (44.5\% vs. 39.6\%) and had emergency admissions (95.1\% vs. 91.9\%). The Southern and West regions documented a higher number of CCE among non-compliant patients. The incidence of major comorbid risk factors such as diabetes, hypertension, obesity and dyslipidemia, peripheral vascular disease, smoking, chronic pulmonary disease, coagulopathy, renal failure, as well as valvular heart disease was also higher in patients with CCE. 


\section{Cureus}

\section{Variable}

Age (years) at hospitalization

Mean age $\pm S D$

18-44

45-64

$\geq 65$

Male

Race

White

African American

Hispanic

Asian or Pacific Islander

Native American

Other

Primary Expected Payer

Medicare

Medicaid

Private including $\mathrm{HMO}$

Self - pay/no charge/others

Median Household Income

0-25th

26-50th

51-75th

76-100th

Non-elective admission

Bed size of hospital

Small

Medium

Large

Location/Teaching Status of Hospital

Rural

Urban - non-teaching

Urban - teaching

Region of Hospital

Northeast

Midwest

South

West
Hospitalizations with Medication Non-compliance $(n=7,453,831)$

No CCE $(n=6,585,834)$

Yes CCE $(n=867,997)$

$51.0 \pm 17.0$

$59.6 \pm 14.7$

$<0.001$

$34.7 \%$

$14.3 \%$

$50.1 \%$

$35.6 \%$

$62.1 \%$

$<0.001$

$<0.001$

$50.5 \%$

$33.1 \%$

$10.9 \%$

$1.7 \%$

$0.8 \%$

$3.0 \%$

$<0.001$

$39.6 \%$

$27.5 \%$

$16.6 \%$

$16.3 \%$

$58.0 \%$

$27.0 \%$

$9.3 \%$

$2.2 \%$

$0.6 \%$

$2.9 \%$

$44.5 \%$

$17.2 \%$

$21.2 \%$

$17.2 \%$

$40.6 \%$

$25.2 \%$

$20.1 \%$

$14.1 \%$

$91.9 \%$

$12.5 \%$

$61.3 \%$

$8.9 \%$

$52.9 \%$
$20.4 \%$

$22.8 \%$

$40.0 \%$

$16.8 \%$
$<0.001$

$<0.001$

$11.2 \%$

$25.1 \%$

$63.7 \%$

$<0.001$

$8.3 \%$

$39.2 \%$

$52.5 \%$

$<0.001$

$<0.001$

$36.9 \%$

$5.5 \%$

$95.1 \%$

$16.7 \%$

$22.1 \%$

$43.3 \%$

$17.9 \%$ 


\section{Cureus}

\begin{tabular}{|c|c|c|c|}
\hline \multicolumn{4}{|l|}{ Comorbidities } \\
\hline Alcohol abuse & $14.1 \%$ & $11.5 \%$ & $<0.001$ \\
\hline Deficiency anemias & $19.5 \%$ & $16.6 \%$ & $<0.001$ \\
\hline Congestive heart failure & $9.2 \%$ & $8.3 \%$ & $<0.001$ \\
\hline Dyslipidemia & $26.6 \%$ & $47.8 \%$ & $<0.001$ \\
\hline Smoking & $41.1 \%$ & $46.6 \%$ & $<0.001$ \\
\hline Chronic pulmonary disease & $21.8 \%$ & $22.6 \%$ & $<0.001$ \\
\hline Coagulopathy & $4.4 \%$ & $4.9 \%$ & $<0.001$ \\
\hline Depression & $11.8 \%$ & $10.2 \%$ & $<0.001$ \\
\hline Diabetes, uncomplicated & $22.7 \%$ & $31.4 \%$ & $<0.001$ \\
\hline Diabetes with chronic complications & $8.3 \%$ & $8.4 \%$ & 0.174 \\
\hline Drug abuse & $18.1 \%$ & $9.8 \%$ & $<0.001$ \\
\hline Hypertension & $50.9 \%$ & $73.7 \%$ & $<0.001$ \\
\hline Hypothyroidism & $8.4 \%$ & $8.4 \%$ & 0.119 \\
\hline Liver disease & $4.9 \%$ & $3.0 \%$ & $<0.001$ \\
\hline Fluid and electrolyte disorders & $27.7 \%$ & $25.7 \%$ & $<0.001$ \\
\hline Obesity & $16.7 \%$ & $19.9 \%$ & $<0.001$ \\
\hline Peripheral vascular disorders & $5.5 \%$ & $8.8 \%$ & $<0.001$ \\
\hline Pulmonary circulation disorders & $1.9 \%$ & $4.4 \%$ & $<0.001$ \\
\hline Renal failure & $14.8 \%$ & $17.6 \%$ & $<0.001$ \\
\hline Valvular heart disease & $2.6 \%$ & $3.5 \%$ & $<0.001$ \\
\hline
\end{tabular}

TABLE 1: Baseline Characteristics of Adult Patients Hospitalized with Medication Non-compliance with vs. Without In-hospital Cardiovascular/Cerebrovascular Events

P-values $<0.05$ indicate statistical significance.

${ }^{\dagger} \mathrm{HMO}=$ Health Maintenance Organization.

CCE=cardiovascular and cerebrovascular events.

Cardiovascular and cerebrovascular events included first or subsequent myocardial infarction, first or subsequent stroke, out-of-hospital cardiac arrest, arrhythmias, and venous thromboembolism.

Figure 1 demonstrates time trends in the prevalence of medication non-compliance among adult patients hospitalized from 2007 to 2014 according to their age group, gender, and race. The study demonstrates a rising trend in each age group, each race of people, and both the genders. Among the age groups, 45-64 years age group had the highest prevalence of medication non-compliance (3.2\% in 2007 to $6.1 \%$ in 2014) followed by $18-44$ years and $\geqslant 65$ years age groups. Similarly, based on the gender, males $(2.9 \%$ in 2007 to 5.5\% in 2014) and among the race groups, African Americans (5.4\% in 2007 to 8.4\% in 2014) had the highest prevalence of medication nonadherence followed by Native American, Hispanic, others, White, and Asian or Pacific Islander. 


\section{Cureus}

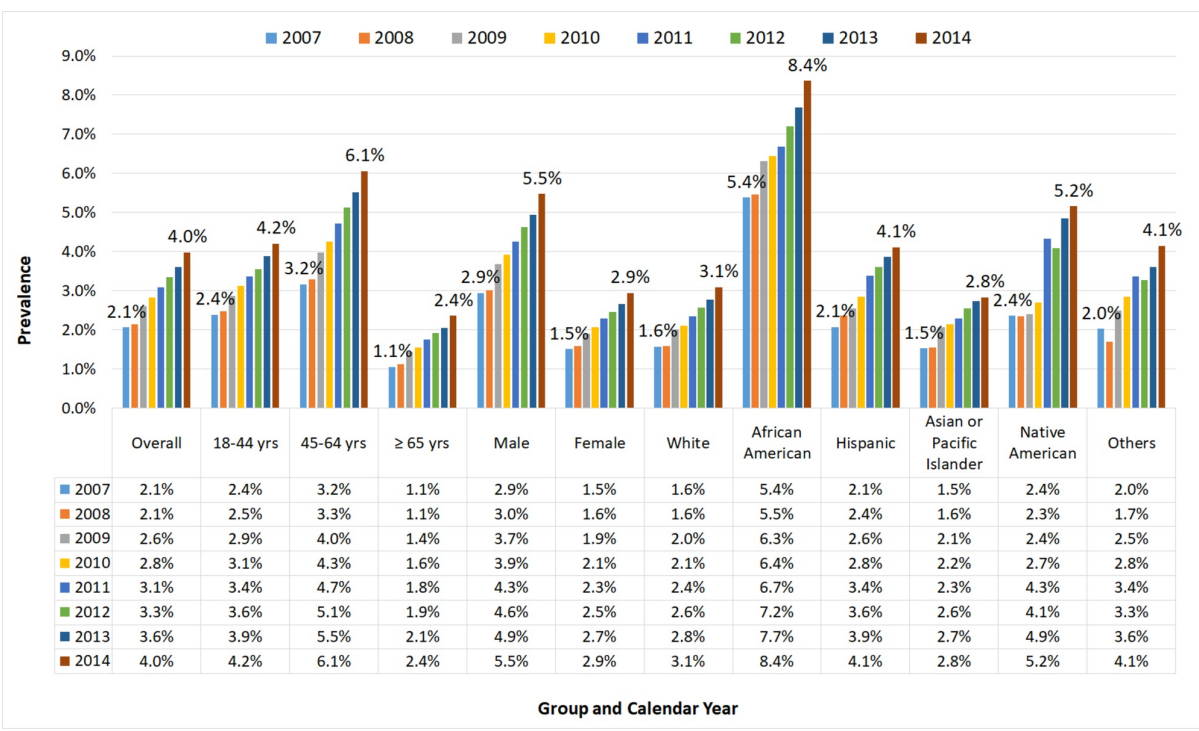

FIGURE 1: Trends in Prevalence of Medication Non-compliance Stratified by Age, Sex, and Race Among Adult Patients Hospitalized from 2007 to 2014

Figure 2 demonstrates in-hospital outcomes as well as mean LOS and hospital charges with or without CCE in patients with medication non-compliance. All-cause in-hospital mortality was significantly higher in patients with CCE compared to without such events (3\% vs. $0.7 \%$ ). In terms of disposition, the non-CCE group had a significantly lower number of routine discharges than patients with CCE (59.4\% vs. $71.1 \%)$, whereas a greater number of transfers were associated with the CCE group (4.4\% vs. $1.8 \%$ transfers to shortterm hospitals and $17.6 \%$ vs. $11.6 \%$ other transfers including skilled nursing facility, intermediate care facility, etc.). Though the mean LOS was equivalent in both the groups, mean hospital charges were considerably higher in non-compliant patients with CCE vs. without CCE ( $\$ 52,740$ vs. $\$ 30,748)$. 


\section{Cureus}

a.

$=$ No CCE $(n=6,585,834) \quad=$ Yes CCE $(n=867,997) \quad p<0.001$

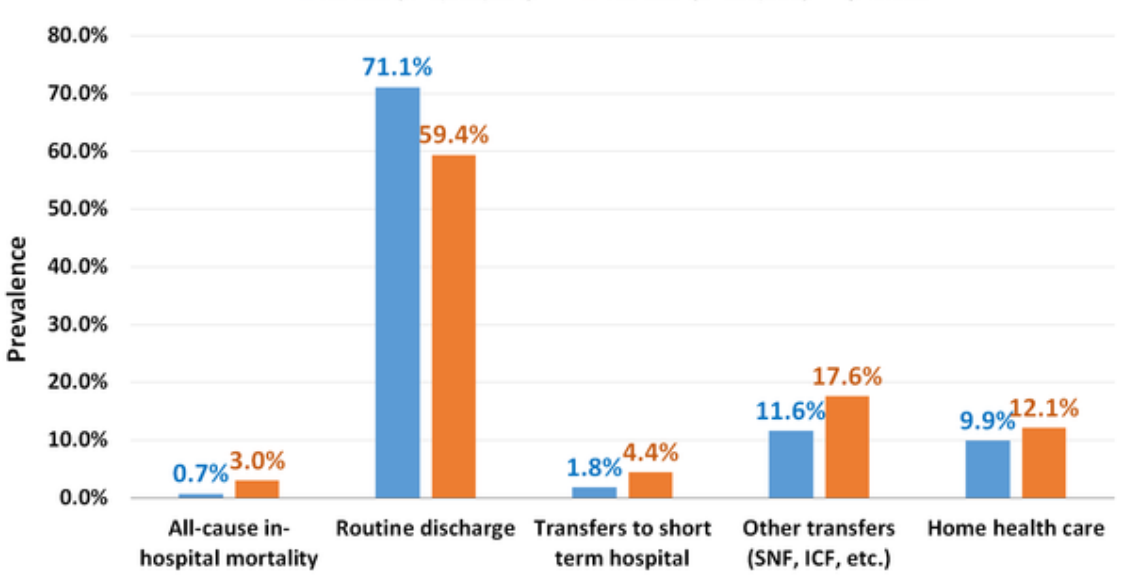

Discharge of Patient

b.

Mean length of stay (days) $\quad$ - Mean hospital charges $\quad \mathrm{p}<0.001$

6.0

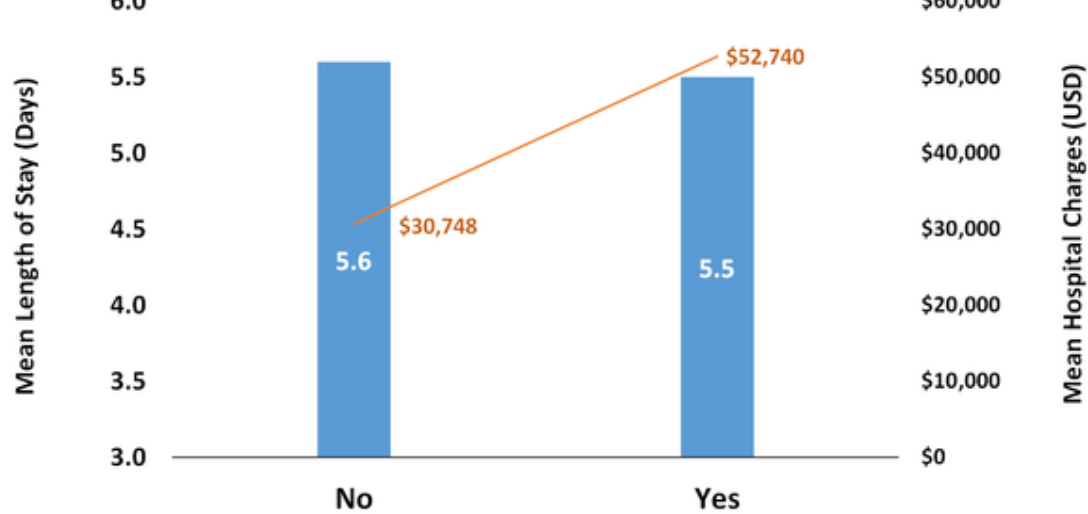

Cardiovascular and Cerebrovascular Events

FIGURE 2: In-hospital Outcomes and Health Care Resource Utilization in Non-compliant Adult Patients with vs. Without In-hospital Cardiovascular and Cerebrovascular Events

a. In-hospital outcomes and disposition in non-compliant patients with vs. without in-hospital cardiovascular and cerebrovascular events.

b. Mean length of stay (days) and hospital charges (United States dollar) in non-compliant patients with vs. without in-hospital cardiovascular and cerebrovascular events.

$\mathrm{CCE}=$ cardiovascular and cerebrovascular events

Cardiovascular or cerebrovascular events included first or subsequent myocardial infarction, first or subsequent stroke, out-of-hospital cardiac arrest, arrhythmias, and venous thromboembolism.

Table 2 demonstrates time trends for in-hospital mortality, cardiovascular and cerebrovascular complications, disposition, mean LOS, as well as hospital charges in hospitalized patients with a history of medication non-compliance from 2007 to 2014. The study demonstrated a rising trend of all-cause inhospital mortality from $0.7 \%$ in 2007 to $1.1 \%$ in 2014. Similarly, over time, prevalence of many CCE also displayed an upward sloping trend whether it was $2 \%$ of first MI in 2007 to $2.5 \%$ in $2014,2.9 \%$ of the first stroke and $0.1 \%$ of consecutive stroke in 2007 to $3 \%$ and $0.6 \%$ in 2014, respectively. Similarly, the proportion of venous thrombosis, out-of-hospital cardiac arrest, and arrhythmia were also higher in 2014 compared to 2007. However, the proportion of patients discharged routinely and transferred to short-term hospital decreased consistently from 2007 to 2014 (62.2\% in 2007 to 56.9\% in 2014). But the trend for 
transfers to other facilities (skilled nursing facility, intermediate care facility, etc) and home health care increased since 2007. Though inconsistently, the mean LOS rose from 5.17 days in 2007 to 5.69 days in 2014 and finally the health care cost also consistently increased from $\$ 36,423$ in 2007 to $\$ 62,341$ in 2014. (For all the aforementioned results, $\mathrm{p}$-value was $<0.001)$.

\begin{tabular}{|c|c|c|c|c|c|c|c|c|c|c|c|}
\hline Outcomes & Overall & 2007 & 2008 & 2009 & 2010 & 2011 & 2012 & 2013 & 2014 & $P_{\text {trend }}$ & Direction \\
\hline All-cause in-hospital mortality & $1.0 \%$ & $0.7 \%$ & $0.8 \%$ & $1.0 \%$ & $0.9 \%$ & $1.0 \%$ & $1.0 \%$ & $1.0 \%$ & $1.1 \%$ & $<0.001$ & $\uparrow$ \\
\hline First myocardial infarction & $2.3 \%$ & $2.0 \%$ & $2.2 \%$ & $2.3 \%$ & $2.2 \%$ & $2.3 \%$ & $2.4 \%$ & $2.4 \%$ & $2.5 \%$ & $<0.001$ & $\uparrow$ \\
\hline First stroke & $2.9 \%$ & $2.9 \%$ & $2.8 \%$ & $2.6 \%$ & $2.9 \%$ & $2.9 \%$ & $2.9 \%$ & $3.0 \%$ & $3.0 \%$ & $<0.001$ & $\uparrow$ \\
\hline Subsequent myocardial infarction & $1.5 \%$ & $1.2 \%$ & $1.3 \%$ & $1.4 \%$ & $1.4 \%$ & $1.6 \%$ & $1.6 \%$ & $1.6 \%$ & $1.6 \%$ & $<0.001$ & $\uparrow$ \\
\hline Subsequent stroke & $0.4 \%$ & $0.1 \%$ & $0.3 \%$ & $0.4 \%$ & $0.5 \%$ & $0.5 \%$ & $0.5 \%$ & $0.6 \%$ & $0.6 \%$ & $<0.001$ & $\uparrow$ \\
\hline Venous thromboembolic events & $2.2 \%$ & $1.9 \%$ & $2.0 \%$ & $2.2 \%$ & $2.1 \%$ & $2.3 \%$ & $2.3 \%$ & $2.3 \%$ & $2.5 \%$ & $<0.001$ & $\uparrow$ \\
\hline Out-of-hospital cardiac arrest & $0.21 \%$ & $0.17 \%$ & $0.17 \%$ & $0.20 \%$ & $0.21 \%$ & $0.22 \%$ & $0.23 \%$ & $0.21 \%$ & $0.25 \%$ & $<0.001$ & $\uparrow$ \\
\hline Arrhythmia & $17.4 \%$ & $13.6 \%$ & $14.7 \%$ & $16.3 \%$ & $16.7 \%$ & $18.1 \%$ & $18.5 \%$ & $18.6 \%$ & $20.0 \%$ & $<0.001$ & $\uparrow$ \\
\hline \multicolumn{12}{|l|}{ Disposition } \\
\hline Routine discharge & $59.4 \%$ & $62.2 \%$ & $63.3 \%$ & $60.6 \%$ & $59.3 \%$ & $58.4 \%$ & $59.5 \%$ & $58.6 \%$ & $56.9 \%$ & $<0.001$ & $\downarrow$ \\
\hline Transfers to short-term hospital & $4.4 \%$ & $5.5 \%$ & $4.6 \%$ & $4.4 \%$ & $4.8 \%$ & $4.6 \%$ & $4.0 \%$ & $3.8 \%$ & $4.0 \%$ & $<0.001$ & $\downarrow$ \\
\hline Other transfers $\left(\mathrm{SNF}^{\dagger}, \mathrm{ICF}^{\ddagger}\right.$, etc.) & $17.6 \%$ & $15.6 \%$ & $15.3 \%$ & $16.9 \%$ & $17.6 \%$ & $18.3 \%$ & $17.5 \%$ & $18.3 \%$ & $19.4 \%$ & $<0.001$ & $\uparrow$ \\
\hline Home health care & $12.1 \%$ & $10.2 \%$ & $10.4 \%$ & $11.2 \%$ & $12.0 \%$ & $12.6 \%$ & $12.8 \%$ & $12.8 \%$ & $13.1 \%$ & $<0.001$ & $\uparrow$ \\
\hline Mean length of stay (days) & 5.52 & 5.17 & 5.13 & 5.63 & 5.64 & 5.56 & 5.48 & 5.56 & 5.69 & $<0.001$ & $\uparrow$ \\
\hline Mean hospital charges & $\$ 52,740$ & $\$ 36,423$ & $\$ 39,437$ & $\$ 48,278$ & $\$ 50,460$ & $\$ 54,274$ & $\$ 55,643$ & $\$ 59,701$ & $\$ 62,341$ & $<0.001$ & $\uparrow$ \\
\hline
\end{tabular}

TABLE 2: Trends in the In-hospital Mortality, Complications, and Health Care Resource Utilization in Hospitalized Adults with Medication Non-compliance

$P_{\text {trend }}$ value $<0.05$ indicates statistical significance.

†SNF=skilled nursing facility,

$\ddagger_{I} \mathrm{CF}=$ intermediate care facility.

$\uparrow$ indicates rising trends and $\downarrow$ indicates declining trends.

\section{Discussion}

Our findings obtained in a study of a large nationwide cohort indicated that nearly $12 \%$ of patients hospitalized with a history of medication non-compliance developed CCE and had suffered higher inhospital mortality, upward trends in all-cause mortality and in-hospital cardiovascular complications, and higher resource utilization as compared to non-compliant patients without CCE. Patients developing CCE had a lower rate of routine discharge and a higher rate of transfers to other facilities and requirement of home health care. The health care cost was also significantly higher in non-compliant patients with CCE. Our study showed increasing trends in the in-hospital mortality and complications related to CCE from 2007 to 2014 .

Adherence is a challenge mainly for chronic diseases, especially during the symptom-free intervals. The consequences of nonadherence are disease mismanagement, progression, and development of complications which lead to increased use of medical resources in the form of frequent health care visits, dependence on health care facilities and hospitalizations, and all these lead to poor outcomes in terms of quality of life, increased mortality, and greater cost of care [14]. In the United States, excess hospitalizations due to medication nonadherence cost approximately $\$ 100$ billion every year [15]. Several studies have found that overall health care costs are much higher for patients with poor adherence as compared to patients with good adherence. A review summarizing the impact of nonadherence on health care cost in several chronic diseases such as diabetes, CVD, and chronic obstructive pulmonary disease concluded that increasing the 
medication adherence significantly reduces the overall health care cost [16]. Our prior analyses have revealed close etiopathological and prognostic link of cardiovascular disorders and outcomes with endocrine, respiratory, or psychiatric disorders which also suggests that medications targeted for one organ system can help prevent overall complications, improve quality of life, and improve long-term prognosis [13, 17-21].

Correspondingly, several studies have described how nonadherence increases individual comorbid diseases or risk factors and in turn, in-hospital mortality. One study by Rasmussen et al. explored the association between drug adherence and mortality in survivors of acute MI which showed that in comparison with the patients with high levels of adherence to statins, the risk of mortality was $25 \%$ higher among patients with poor adherence [22]. Similarly, one study assessing the risk of fatal stroke associated with nonadherence to statin and antihypertensive therapy derived that individuals with hypercholesterolemia and hypertension who failed to take their prescribed medications experienced a substantially increased (up to twice) risk of fatal stroke [23]. Studies have established the demographics of patients more frequently found to be linked to medication non-compliance. As one leap forward, we studied the baseline characteristics and comorbidities among non-compliant patients with CCE and compared it to patients without CCE to help clinicians to understand the demographics of the nonadherent high-risk patient population. We observed that the patients with CCE were older, more frequently white, men and had a higher prevalence of comorbidities. Non-compliant patients developing CCE had worse outcomes, underwent excessive health care utilization (transfers to short-term hospitals, intermediate care, and skilled nursing facilities, home health care) and in turn, the higher cost of care per admission. Our findings from a much larger patient population linking the medication nonadherence to in-hospital outcomes expand the literature on nonadherence and emphasize the importance of medication adherence in clinical practice.

The challenge with medication nonadherence is that there does not exist a particular parameter to measure it and it is invisible to patients, families, and health care providers. To deal with the emerging concerns pertinent to nonadherence, we should identify the barriers related to nonadherence which come under the categories of patient-related factors which include poor knowledge (most common predictor), lack of motivation, depression, denial, and cultural beliefs; treatment-related factors which include the type of illness, complexity of treatment, side effects, cost, and others most importantly physician-patient relationship [24, 25]. Then comes the methods of measuring nonadherence such as directly observed therapy, identifying the level of medicine in the blood (most reliable); indirect methods such as patient questionnaires, pill counts, clinical response, electronic monitors, patient diary, and many more [26]. Even after incorporating different interventions to increase the drug adherence including prescribing generic medications, simplifying the schedule, patients' health coaching, close follow-up, frequent communication with treating physicians, and using mobile health care applications [26-29], there are number of populationbased studies showing the decreasing compliance rate for multiple medications in the short term and long term [30]. The trends in hospitalizations with worsened outcomes heightened complications, and health care costs related to medication nonadherence were consistently evident from our study results.

\section{Limitations}

This study has several limitations. The current analysis does not reveal nonadherence to a specific group of medication or disease. Because of the retrospective nature of the study, it represents only an association and not causation. Over- or underreporting of the study population could be likely secondary to administrative coding errors in the NIS database. Despite a few limitations, a large sample size increases the generalizability of the study findings.

\section{Conclusions}

In this population-based nationwide analysis, we observed concerning trends in the prevalence of medication non-compliance and related CCE among hospitalized adults from 2007 to 2014 which was associated with higher in-hospital mortality, health care utilization, and cost. This study highlights the role of health care practitioners in screening older patients with chronic conditions for poor medication compliance and incorporating a more robust and collaborative approach which may possibly make a difference in clinical medicine on a global level.

\section{Appendices}




\section{Cureus}

\begin{tabular}{|c|c|}
\hline Disease/Event/Outcome & ICD-9 CM/CCS Code \\
\hline $\begin{array}{l}\text { Acute myocardial } \\
\text { infarction }\end{array}$ & $\operatorname{ccs} 100$ \\
\hline Arrhythmia & $\operatorname{CCS} 106$ \\
\hline $\begin{array}{l}\text { Acute cerebrovascular } \\
\text { disease/stroke }\end{array}$ & CCS 109 \\
\hline $\begin{array}{l}\text { Previous myocardial } \\
\text { infarction }\end{array}$ & ICD-9 412 \\
\hline $\begin{array}{l}\text { Previous transient } \\
\text { ischemic attack/stroke }\end{array}$ & ICD-9 V12.54 \\
\hline $\begin{array}{l}\text { Venous } \\
\text { thromboembolism }\end{array}$ & ICD-9 415.1x, 451.1x, 451.2, 451.8x, 451.9, 453.2, 453.4x, 453.8x, 453.9 \\
\hline $\begin{array}{l}\text { Subsequent myocardial } \\
\text { infarction }\end{array}$ & $\begin{array}{l}\text { Primary discharge diagnosis of acute myocardial infarction (CCS 100) with any of secondary discharge } \\
\text { diagnoses of previous myocardial infarction (ICD-9 412) }\end{array}$ \\
\hline Subsequent stroke & $\begin{array}{l}\text { Primary discharge diagnosis of acute cerebrovascular disease (CCS 109) with any of secondary } \\
\text { discharge diagnoses of previous transient ischemic attack/stroke (ICD-9 V12.54) }\end{array}$ \\
\hline $\begin{array}{l}\text { Percutaneous coronary } \\
\text { intervention }\end{array}$ & $\operatorname{ccs} 45$ \\
\hline $\begin{array}{l}\text { Coronary artery bypass } \\
\text { grafting }\end{array}$ & $\operatorname{ccs} 44$ \\
\hline
\end{tabular}

\section{TABLE 3: International Classification of Diseases, 9th Revision, Clinical Modification (ICD-9-}

CM)/Clinical Classifications Software (CCS) Codes Used to Identify Cardiovascular Events and Outcomes

CCS is based on the International Classification of Diseases, 9th Revision, Clinical Modification (ICD-9-CM), and a uniform and standardized coding system. The ICD-9-CM's multitude of codes - over 14,000 diagnosis codes and 3,900 procedure codes - are collapsed into a smaller number of clinically meaningful categories that are sometimes more useful for presenting descriptive statistics than are individual ICD-9-CM codes.

Source: HCUP CCS. Healthcare Cost and Utilization Project (HCUP). March 2017. Agency for Healthcare Research and Quality, Rockville, MD. www.hcup-us.ahrq.gov/toolssoftware/ccs/ccs.jsp. Accessed July 22, 2019.

\section{Additional Information \\ Disclosures}

Human subjects: Consent was obtained by all participants in this study. Animal subjects: All authors have confirmed that this study did not involve animal subjects or tissue. Conflicts of interest: In compliance with the ICMJE uniform disclosure form, all authors declare the following: Payment/services info: All authors have declared that no financial support was received from any organization for the submitted work. Financial relationships: All authors have declared that they have no financial relationships at present or within the previous three years with any organizations that might have an interest in the submitted work. Other relationships: All authors have declared that there are no other relationships or activities that could appear to have influenced the submitted work.

\section{References}

1. World Health Organization: Adherence to long-term therapies: evidence for action. Noncommunicable diseases and mental health adherence to long-term therapies project. 2003, Accessed: June 9, 2014: http://www.who.int/chp/knowledge/publications/adherence_introduction.pdf.

2. Naderi SH, Bestwick JP, Wald DS: Adherence to drugs that prevent cardiovascular disease: meta-analysis on 376,162 patients. Am J Med. 2012, 125:882-887. 10.1016/j.amjmed.2011.12.013

3. Ma TT, Wong ICK, Man KKC, et al.: Effect of evidence-based therapy for secondary prevention of cardiovascular disease: systematic review and meta-analysis. PLoS One. 2019, 14:0210988. 10.1371/journal.pone.0210988

4. Xu T, Yu X, Ou S, et al.: Adherence to antihypertensive medications and stroke risk: a dose-response metaanalysis. J Am Heart Assoc. 2017, 6:e006371. 10.1161/jaha.117.006371

5. Ho PM, Magid DJ, Shetterly SM, et al.: Medication nonadherence is associated with a broad range of adverse 
outcomes in patients with coronary artery disease. Am Heart J. 2008, 155:772-779. 10.1016/j.ahj.2007.12.011

6. Baroletti S, Dell'Orfano H: Medication adherence in cardiovascular disease . Circulation. 2010, 121:14551458. 10.1161/circulationaha.109.904003

7. Bansilal S, Castellano JM, Garrido E, et al.: Assessing the impact of medication adherence on long-term cardiovascular outcomes. J Am Coll Cardiol. 2016, 68:789-801. 10.1016/j.jacc.2016.06.005

8. Bitton A, Choudhry NK, Matlin OS, Swanton K, Shrank WH: The impact of medication adherence on coronary artery disease costs and outcomes: a systematic review. Am J Med. 2013, 126:357-357. 10.1016/j.amjmed.2012.09.004

9. Cutler RL, Fernandez-Llimos F, Frommer M, Benrimoj C, Garcia-Cardenas V: Economic impact of medication non-adherence by disease groups: a systematic review. BMJ Open. 2018, 8:016982. 10.1136/bmjopen-2017-016982

10. Gosmanova EO, Lu JL, Streja E, Cushman WC, Kalantar-Zadeh K, Kovesdy CP: Association of medical treatment nonadherence with all-cause mortality in newly treated hypertensive US veterans. Hypertension. 2014, 64:951-957. 10.1161/hypertensionaha.114.03805

11. Desai R, Patel U, Rupareliya C, et al.: Impact of cocaine use on acute ischemic stroke patients: insights from Nationwide Inpatient Sample in the United States. Cureus. 2017, 9:1536. 10.7759/cureus.1536

12. Desai R, Patel U, Sharma S, et al.: Recreational marijuana use and acute myocardial infarction: insights from Nationwide Inpatient Sample in the United States. Cureus. 2017, 9:1816. 10.7759/cureus.1816

13. Desai R, Singh S, Baikpour M, et al.: Does obesity affect the outcomes in takotsubo cardiomyopathy? Analysis of the Nationwide Inpatient Sample database, 2010-2014. Clin Cardiol. 2018, 41:1028-1034. 10.1002/clc.22999

14. Jimmy B, Jose J: Patient medication adherence: measures in daily practice. Oman Med J. 2011, 26:155-159. 10.5001/omj.2011.38

15. New England Healthcare Institute: Thinking outside the pillbox: a system-wide approach to improving patient medication adherence for chronic disease. NEHI Research Brief. August 2009, Accessed: July 2019: https://www.nehi.net/writable/publication_files/file/pa_issue_brief_final.pdf.

16. Iuga AO, McGuire MJ: Adherence and health care costs. Risk Manag Healthc Policy. 2014, 7:35-44. 10.2147/rmhp.s19801

17. Desai R, Patel U, Singh S, et al.: The burden and impact of arrhythmia in chronic obstructive pulmonary disease: insights from the National Inpatient Sample. Int J Cardiol. 2019, 281:49-55. 10.1016/j.ijcard.2019.01.074

18. Desai R, Rupareliya C, Patel U, et al.: Burden of arrhythmias in epilepsy patients: a nationwide inpatient analysis of 1.4 million hospitalizations in the United States. Cureus. 2017, 9:1550. 10.7759/cureus.1550

19. Desai R, Goyal H, Kumar G, Sachdeva R: Impact of hypothyroidism on outcomes of percutaneous coronary intervention with coronary atherectomy for calcified coronary lesions: a propensity-matched analysis. Cureus. 2018, 10:2600. 10.7759/cureus.2600

20. Desai R, Singh S, Patel K, Fong HK, Kumar G, Sachdeva R: The prevalence of psychiatric disorders in sudden cardiac arrest survivors: a 5-year nationwide inpatient analysis. Resuscitation. 2019, 136:131-135. 10.1016/j.resuscitation.2019.01.035

21. Desai R, Kakumani K, Fong HK, et al.: The burden of cardiac arrhythmias in sarcoidosis: a population-based inpatient analysis. Ann Transl Med. 2018, 6:330. 10.21037/atm.2018.07.33

22. Rasmussen JN, Chong A, Alter DA: Relationship between adherence to evidence-based pharmacotherapy and long-term mortality after acute myocardial infarction. JAMA. 2007, 297:177-186. 10.1001/jama.297.2.177

23. Herttua K, Martikainen P, Batty GD, Kivimaki M: Poor adherence to statin and antihypertensive therapies as risk factors for fatal stroke. J Am Coll Cardiol. 2016, 67:1507-1515. 10.1016/j.jacc.2016.01.044

24. Kleinsinger F: The unmet challenge of medication nonadherence . Perm J. 2018, 22:18-033. 10.7812/tpp/18033

25. Bowry AD, Shrank WH, Lee JL, Stedman M, Choudhry NK: A systematic review of adherence to cardiovascular medications in resource-limited settings. J Gen Intern Med. 2011, 26:1479-1491. 10.1007/s11606-011-1825-3

26. Osterberg L, Blaschke T: Adherence to medication. N Engl J Med. 2005, 353:487-497. 10.1056/NEJMra050100

27. McCormick JB, Green MJ, Shapiro D: Medication nonadherence: there's an app for that! . Mayo Clin Proc. 2018, 93:1346-1350. 10.1016/j.mayocp.2018.05.029

28. Shrank WH, Hoang T, Ettner SL, et al.: The implications of choice: prescribing generic or preferred pharmaceuticals improves medication adherence for chronic conditions. Arch Intern Med. 2006, 166:332337. 10.1001/archinte.166.3.332

29. Thom DH, Willard-Grace R, Hessler D, et al.: The impact of health coaching on medication adherence in patients with poorly controlled diabetes, hypertension, and/or hyperlipidemia: a randomized controlled trial. J Am Board Fam Med. 2015, 28:38-45. 10.3122/jabfm.2015.01.140123

30. Newby LK, LaPointe NM, Chen AY, et al.: Long-term adherence to evidence-based secondary prevention therapies in coronary artery disease. Circulation. 2006, 113:203-212. 10.1161/circulationaha.105.505636 